\title{
The Clinical Significance of Changes in Serum New Cytokine CYTLI in Patients with Knee Osteoarthritis
}

\author{
Jian-Sheng Sun ${ }^{1, *}$ \\ Wei-Wei Wang ${ }^{1, *}$ \\ Hong-Kai Lian ${ }^{2}$ \\ 'Department of Orthopedics, The \\ Second Affiliated Hospital of Zhengzhou \\ University, Zhengzhou, 450000, Henan, \\ People's Republic of China; ${ }^{2}$ Department \\ of Orthopedics, Zhengzhou Central \\ Hospital, Zhengzhou, 450000, Henan, \\ People's Republic of China
}

*These authors contributed equally to this work
Correspondence: Hong-Kai Lian Department of Orthopedics, Zhengzhou Central Hospital, No. 195, Tongbai Road Middle Section, Zhongyuan District, Zhengzhou, 450000, Henan, People's Republic of China

$\mathrm{Tel}+86$ I8837I89223

Email lianhk@tom.com
Objective: The present study aims to investigate the clinical significance of changes in the expression of new cytokine-like 1 (CYTL1) in the serum of patients with knee osteoarthritis (KOA).

Methods: A total of 182 patients with KOA, including 84 males and 98 females aged 39-86 with an average age of $66.4 \pm 9.7$ and an average body mass index (BMI) of $24.9 \pm 2.4 \mathrm{~kg} /$ $\mathrm{m}^{2}$, were enrolled in the study. The patients were divided into three subgroups: the grade II subgroup $(n=23)$, grade III subgroup $(n=63)$, and grade IV subgroup $(n=96)$ based on severity, as calculated by the Kellgren and Lawrence (K\&L) classification system. In addition, 152 volunteers from our health center who came in for physical examination were selected as the control group, including 70 males and 82 females aged 37-82 with an average age of $63.4 \pm 9.5$ and an average BMI of $24.8 \pm 2.2 \mathrm{~kg} / \mathrm{m}^{2}$. An enzyme-linked immunosorbent assay was adopted to detect the serum CYTL1 levels, and the correlation between CYTL1 and the severity of KOA was analyzed.

Results: The serum level of CYTL1 was significantly lower in the KOA group than in the control group $(\mathrm{P}<0.05)$. In the KOA group, the difference in the serum level of CYTL1 was statistically significant between the subgroups and decreased significantly with an increase in the severity of the disease $(\mathrm{F}=54.826, \mathrm{P}<0.001)$. Therefore, the serum level of CYTL1 was correlated with the severity of the disease, as determined by the K\&L classification system $(\mathrm{r}=-0.613, \mathrm{P}<0.001)$.

Conclusion: The serum levels of CYTL1 are strongly correlated with the severity of the disease in patients with KOA and could be a new therapeutic target for KOA.

Keywords: osteoarthritis, serum, new cytokine-like 1, correlation

\section{Introduction}

Osteoarthritis (OA) is the most common chronic joint disease and is the leading cause of mobility problems in older people. The prevalence of OA increases with age, affecting most people over the age of $65 .{ }^{1,2}$ The main clinical symptoms of OA include chronic pain, joint instability, stiffness, joint deformity, and radiographic narrowing of the joint space. ${ }^{3,4}$ Although several risk factors associated with OA have been proposed, including genetic predisposition, aging, obesity, and joint misalignment, its pathogenesis remains unclear. ${ }^{5,6}$ Currently, the standard method for identifying the severity of bone and joint injury is the Kellgren and Lawrence (K\&L) classification system, which is based on measuring the width of the joint space on plain radiographs. ${ }^{7}$ The detection of early metabolic lesions in joints 
before developing imaging changes in patients is clinically important for identification and individualized treatment in high-risk patients. New soluble circulating markers need to be identified to meet these objectives. Cytokine-like 1 (CYTL1) is a novel autocrine regulator that is specifically and highly expressed in the chondrocytes and is involved in the formation and development of cartilage and the occurrence and progression of arthritis. ${ }^{8}$ The correlation between the serum concentration of CYTL1 and the severity of OA has been poorly investigated. In the present study, we aim to measure the serum levels of CYTL1 in healthy controls and patients with $\mathrm{OA}$ and analyze the correlation between the serum levels of CYTL1 and the severity of OA. We intend to provide a new means for the early identification of individuals at high risk of articular cartilage destruction and plan to adopt CYTL1 as a new therapeutic target for OA.

\section{Methods}

\section{General Characteristics}

A total of 182 patients with knee OA (KOA) who were admitted to our hospital between October 2019 and November 2020 were selected as subjects for the present study. All cases met the diagnostic criteria of the Guidelines for Osteoarthritis Treatment issued by the Chinese Medical Association Orthopedic Branch. ${ }^{9}$ The subjects consisted of 84 males and 98 females aged 3986 with an average age of $66.2 \pm 9.8$ and an average body mass index (BMI) of $24.9 \pm 2.4 \mathrm{~kg} / \mathrm{m}^{2}$. The disease duration ranged from two to six years. K\&L grading was conducted according to the bone and joint radiographs of the subjects. The subjects were divided into groups based on this grade: (1) grade II: obvious bony bulge but without the involvement of the joint space $(\mathrm{n}=23)$; (2) grade III: moderate narrowing of the joint space $(\mathrm{n}=63)$; and (3) grade IV: significant narrowing of the joint space and subchondral bone sclerosis $(\mathrm{n}=96)$. The exclusion criteria were as follows: (1) patients with a history of surgery or knee trauma; (2) patients with rheumatoid arthritis, ankylosing spondylitis, tumor or tuberculosis of the knee joint, or severe osteoporosis; (3) patients with coagulation disorders or who were on anticoagulants; (4) patients with other sites of osteoarthritis; and (5) patients with serious medical diseases or psychiatric disorders. The ongoing medication of all patients with KOA was celecoxib. In addition to these subjects, during the same period, 152 age- and gender-matched healthy subjects who came for a physical examination at our hospital were selected for the control group. This group consisted of 70 males and 82 females aged 37-82 with an average age of $65.0 \pm 9.5$ and an average BMI of $24.8 \pm 2.2 \mathrm{~kg} / \mathrm{m}^{2}$. The control subjects had no inflammatory diseases or other sites of osteoarthritis, according to their medical records. The present study was approved by the Ethics Committee of Zhengzhou Central Hospital and conducted in accordance with the Declaration of Helsinki. Each patient signed informed consent.

\section{Enzyme-Linked Immunosorbent Assay (ELISA) Detection}

For the detection of the indicators, $5 \mathrm{~mL}$ of venous blood was drawn from the patients and the healthy subjects in the early morning under a fasting condition. Samples that were measured within five days were stored at $4{ }^{\circ} \mathrm{C}$, while those measured over a week were stored at $-20^{\circ} \mathrm{C}$. The serum was separated by centrifugation at $3000 \mathrm{rpm}$ for $10 \mathrm{~min}$ with a centrifugation radius of $10 \mathrm{~cm}$ within 30 min. The serum concentration of CYTL1 was measured by the ELISA using the ELX800 enzyme marker produced by BioTek Instruments, USA. The kit was purchased from the Shanghai Enzyme Linkage Biotechnology Co., and the assay was conducted according to the instructions.

\section{Statistical Processing}

The SPSS 20.0 software was adopted for statistical analysis, and the measurement data were expressed as $\bar{x} \pm s$. The comparison of the serum concentration of CYTL1 between patients with KOA and the control group was performed using the $t$-test and between the different groups of patients with KOA using the one-way ANOVA test. The correlation between the serum concentration of CYTL1 and the severity of KOA was analyzed using the Spearman correlation analysis. $\mathrm{P}<0.05$ was considered statistically significant.

\section{Results}

The Comparison of the General Characteristics Between the Two Groups

There were no statistically significant differences in age, gender composition, or BMI between the KOA group and the control group $(\mathrm{P}>0.05)$. The serum level of CYTL1 in the KOA group was significantly lower than in the control group ( $<<0.05$; see Table 1). 
Table I The Comparison of the General Characteristics Between the Two Groups

\begin{tabular}{|l|c|c|c|c|c|}
\hline Group & $\mathbf{n}$ & Age (Year) & Gender (Female/Male) & BMI (kg/m $\mathbf{2}^{\mathbf{2}}$ & CYTLI (ng/mL) \\
\hline KOA group & 182 & $66.2 \pm 9.8$ & $98 / 84$ & $24.9 \pm 2.4$ & $305.0 \pm 58.8$ \\
Control group & 152 & $65.0 \pm 9.5$ & $82 / 70$ & $24.8 \pm 2.2$ & $402.1 \pm 36.5$ \\
P & & $>0.05$ & $>0.05$ & $>0.05$ & 0.000 \\
\hline
\end{tabular}

Abbreviations: BMI, body mass index; CYTLI, cytokine-like I.

Table 2 The Serum Levels of CYTLI in the Patients $(\overline{\mathrm{x}} \pm \mathrm{s})$

\begin{tabular}{|l|c|c|c|}
\hline & Grade II & Grade III & Grade IV \\
\hline $\mathrm{n}$ & 23 & 63 & 96 \\
CYTLI $(\mathrm{ng} / \mathrm{mL})$ & $383.9 \pm 54.8$ & $321.8 \pm 46.2$ & $276.2 \pm 45.2$ \\
\hline
\end{tabular}

\section{Comparison of the Differences in Serum Levels of CYTLI Among the Subgroups of KOA}

As shown in Table 2, the level of CYTL1 was $383.9 \pm$ $54.8 \mathrm{ng} / \mathrm{mL}$ in patients in the grade II subgroup, 321.8 $\pm 46.2 \mathrm{ng} / \mathrm{mL}$ in the grade III subgroup, and $276.2 \pm$ $45.2 \mathrm{ng} / \mathrm{mL}$ in the grade IV subgroup. The differences in the serum levels of CYTL1 between the KOA subgroups were statistically significant $(\mathrm{F}=54.826, \mathrm{P}<$ $0.001)$.
The correlation between the serum levels of CYTL1 and the severity of KOA (see Figure 1).

\section{Discussion}

$\mathrm{OA}$ is a chronic degenerative joint disease characterized by the degeneration of the articular cartilage, sclerosis of the subchondral bone, formation of bone fragments, and inflammation of the synovial membrane. ${ }^{10}$ In addition, studies have revealed aggressive angiogenesis, the key roles of the pro-inflammatory secretory molecules, and the chondrocyte dedifferentiation in the pathophysiology of OA. ${ }^{11-13}$

CYTL1 is a secreted protein predicted to have the structural characteristics of the hematopoietic cytokines and interleukins and may have immunobiological functions. ${ }^{14}$ CYTL1 is known to regulate the chondrogenic

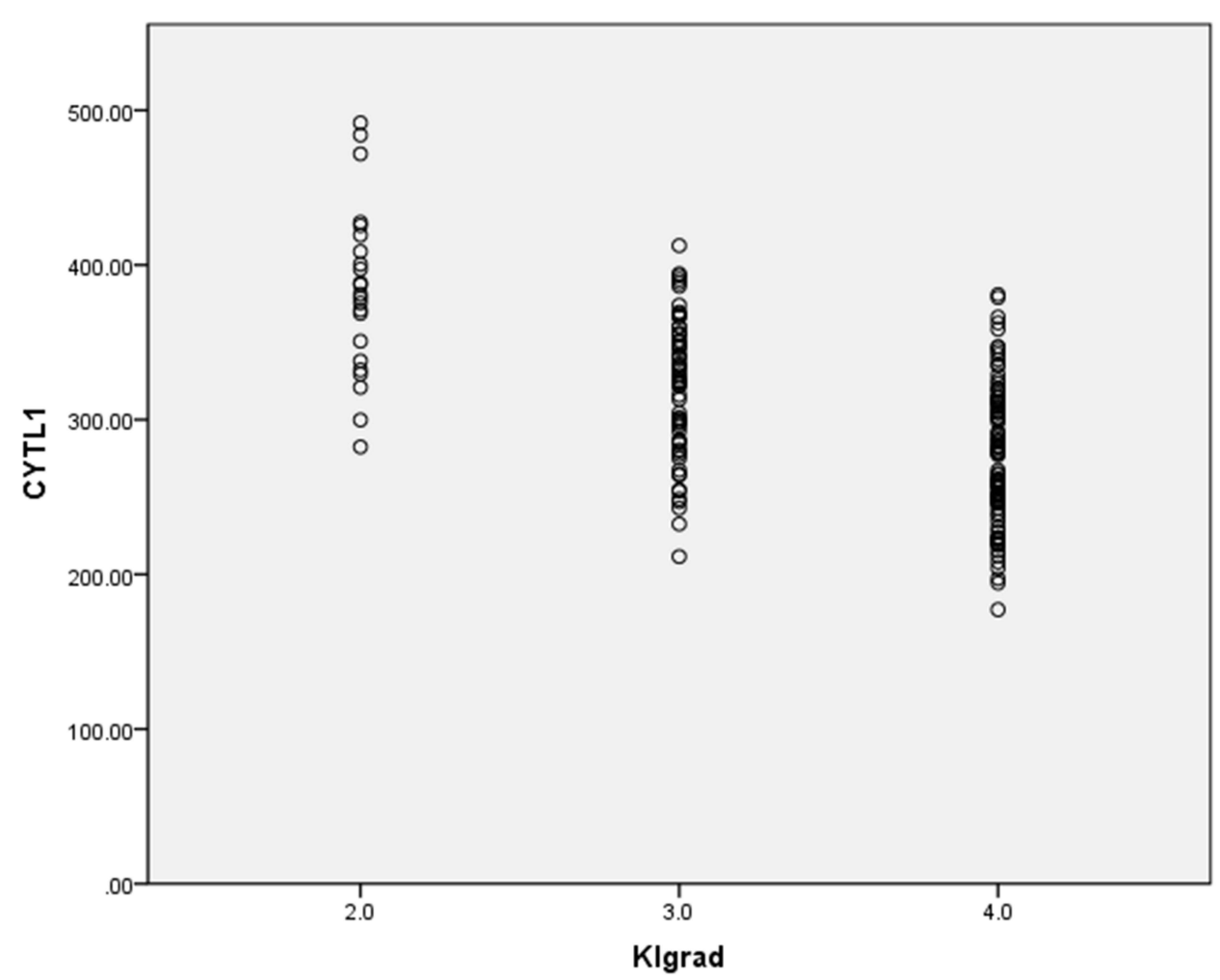

Figure I The negative correlation between the serum levels of CYTLI and the $K L$ grading in the $O A$ group $(r=-0.613, P<0.00 \mathrm{I})$. 
differentiation of the mesenchymal cells and increase the expression of interleukin-1 during cartilage formation. ${ }^{15}$ In addition, CYTL1 appears to have a strong pro-angiogenic effect, which is comparable to the vascular endothelial growth factor $\mathrm{A}$ and has chemotactic activity on monocytes and macrophages via the $\mathrm{CCR} 2 \mathrm{~B}$ receptor. The expression appears to be downregulated during the progression of OA. ${ }^{16-18}$ CYTL1 was originally found to be expressed in the $\mathrm{CD}_{3} 4^{+}$bone marrow and umbilical cord blood cells in the human population. Recently, CYTL1 has been reported to be expressed by the chondrocytes, suggesting a role for CYTL1 in chondrogenesis and endochondral homeostasis. ${ }^{14,19,20}$ CYTL1 was identified as being differentially expressed in post-traumatic osteoarthritis (PTOA), suggesting that CYTL1 may be a potential target for early PTOA-specific intervention and further suggesting a role for CYTL1 in the development of OA. ${ }^{18}$ However, the expression of CYTL1 in the serum of patients remains unclear. Therefore, we investigated the difference in the expression of CYTL1 in the serum of healthy subjects and patients with KOA and between groups of patients with KOA of varying degrees of severity to identify CYTL1 as a potential target for intervention during the progression of KOA.

In the present study, it was found that the serum level of CYTL1 in the KOA group was significantly lower than in the control group, while the serum level of CYTL1 decreased significantly with an increase in the severity of KOA $(\mathrm{P}<0.05)$. The Spearman correlation analysis showed that the serum level of CYTL1 was correlated with the severity of KOA $(r=-0.613, \mathrm{P}<0.001)$, suggesting that CYTL1 may be involved in the development of KOA. It was also found that CYTL1 was mainly expressed in the chondrocytes and cartilage. ${ }^{8,15}$ The exogenous addition of CYTL1 may induce the chondrogenic differentiation of the mesenchymal cells via microcluster culture without affecting the hypertrophic maturation of the chondrocyte. $^{15}$ The chondrogenic effect of CYTL1, which was achieved through the induction of the transcriptional activity of SOX9 and the expression of insulin-like growth factor $1,{ }^{15}$ supports the idea of the involvement of CYTL1 in the development of KOA in the present study.

The limitations of the present study were as follows. First, the origin of the serum CYTL1 in patients with KOA remains unknown, and the question of whether it originates from the diseased knee cartilage needs to be further investigated by pathological biopsy. Second, the clinical knee scores and quality of life of these patients were not assessed. Third, the sample size was small, and the sample was limited to serum. Therefore, there is a need to collect both the joint fluid and the serum and to expand the sample size. Finally, we did not compare the serum levels of CYTL1 with the synovial fluid levels. The relationship between the mechanism of CYTL1 and the articular cartilage needs to be further confirmed in animal experiments.

In summary, the abnormally low serum levels of CYTL1 in patients with KOA were closely correlated with the progression of the disease and, therefore, could be used as a serum biomarker to monitor severity during the progression of the disease. CYTL1 is also worthy of further research as a new therapeutic target for OA. In addition, longitudinal studies are needed to investigate the effect of CYTL1 on the radiological progression of the disease.

\section{Funding}

This study was supported by Key Medical Science and Technology Program of Henan Province (jointly built) [NO.LHGJ20191059].

\section{Disclosure}

The authors declare that they have no competing interests.

\section{References}

1. Dahaghin S, Bierma-Zeinstra SM, Ginai AZ, Pols HA, Hazes JM, Koes BW. Prevalence and pattern of radiographic hand osteoarthritis and association with pain and disability (the Rotterdam study). Ann Rheum Dis. 2005;64:682-687. doi:10.1136/ard.2004.023564

2. Oliveria SA, Felson DT, Reed JI, Cirillo PA, Walker AM. Incidence of symptomatic hand, hip, and knee osteoarthritis among patients in a health maintenance organization. Arthritis Rheum. 1995;38: 1134-1141. doi:10.1002/art.1780380817

3. Felson DT. Clinical practice. Osteoarthritis of the knee. $N$ Engl J Med. 2006;354:841-848. doi:10.1056/NEJMcp051726

4. Goldring MB, Goldring SR. Osteoarthritis. J Cell Physiol. 2007; 213:626-634. doi: $10.1002 /$ jcp. 21258

5. Rai MF, Sandell LJ. Inflammatory mediators: tracing links between obesity and osteoarthritis. Crit Rev Eukaryot Gene Expr. 2011;21:131-142. doi:10.1615/CritRevEukarGeneExpr.v21.i2.30

6. Mobasheri A. Osteoarthritis year 2012 in review: biomarkers. Osteoarthr Cartil. 2012;20:1451-1464. doi:10.1016/j.joca.2012.07. 009

7. Liu B, Gao ZB, Zhao LL. [Association of TWEAK level in serum and synovia with X-ray severity in knee osteoarthritis patients]. Chin J Tradit Med Traumatol Orthop. 2017;25:18-21. Chinese.

8. Jeon J, Oh H, Lee G, et al. Cytokine-like 1 knock-out mice (Cytl1-/-) show normal cartilage and bone development but exhibit augmented osteoarthritic cartilage destruction. J Biol Chem. 2011;286:27 206-27213. doi:10.1074/jbc.M111.218065

9. Joint Surgery Group of Chinese Medical Association Osteology Society Branch. [Clinical guidelines for osteoarthritis (2018 edition)]. Chin J Orthop. 2018;38:705-715. Chinese.

10. Charlier E, Deroyer C, Ciregia F, et al. Chondrocyte dedifferentiation and osteoarthritis (OA). Biochem Pharmacol. 2019;165:49-65. 
11. Vadalà G, Russo F, Musumeci M, Giacalone A, Papalia R, Denaro V. Targeting VEGF-A in cartilage repair and regeneration: state of the art and perspectives. J Biol Regul Homeost Agents. 2018;32:217-224.

12. Kapoor M, Martel-Pelletier J, Lajeunesse D, Pelletier JP, Fahmi H. Role of proinflammatory cytokines in the pathophysiology of osteoarthritis. Nat Rev Rheumatol. 2011;7:33-42. doi:10.1038/nrrheum.2010.196

13. Deroyer C, Charlier E, Neuville S, et al. CEMIP (KIAA1199) induces a fibrosis-like process in osteoarthritic chondrocytes. Cell Death Dis. 2019;10:103. doi:10.1038/s41419-019-1377-8

14. Liu X, Rapp N, Deans R, Cheng L. Molecular cloning and chromosomal mapping of a candidate cytokine gene selectively expressed in human CD34+ cells. Genomics. 2000;65:283-292. doi:10.1006/geno. 2000.6170

15. Kim JS, Ryoo ZY, Chun JS. Cytokine-like 1 (Cytl1) regulates the chondrogenesis of mesenchymal cells. J Biol Chem. 2007;282: 29359-29367. doi:10.1074/jbc.M700965200
16. Wang X, Li T, Wang W, et al. Cytokine-like 1 chemoattracts monocytes/macrophages via CCR2. J Immunol. 2016;196:4090-4099. doi:10.4049/jimmunol.1501908

17. Schneller D, Hofer-Warbinek R, Sturtzel C, et al. Cytokine-like 1 is a novel proangiogenic factor secreted by and mediating functions of endothelial progenitor cells. Circ Res. 2019;124:243-255. doi:10.1161/CIRCRESAHA.118.313645

18. Sieker JT, Proffen BL, Waller KA, et al. Transcriptional profiling of articular cartilage in a porcine model of early post-traumatic osteoarthritis. J Orthop Res. 2018;36:318-329. doi:10.1002/jor.23876

19. Ai Z, Jing W, Fang L, Shen W. Cytokine-like protein 1(Cytl1): a potential molecular mediator in embryo implantation. PLoS One. 2016;11(1):e0147424. doi:10.1371/journal.pone.0147424

20. Chao C, Joyce-Shaikh B, Grein J, et al. 17 prevents inflammatory arthritis and associated joint destruction in mice. PLoS One. 2011;6: e22256.

\section{Publish your work in this journal}

The International Journal of General Medicine is an international, peer-reviewed open-access journal that focuses on general and internal medicine, pathogenesis, epidemiology, diagnosis, monitoring and treatment protocols. The journal is characterized by the rapid reporting of reviews, original research and clinical studies across all disease areas. The manuscript management system is completely online and includes a very quick and fair peer-review system, which is all easy to use. Visit http://www.dovepress.com/ testimonials.php to read real quotes from published authors. 